\title{
High-quality draft genome sequence of Ensifer meliloti Mlalz-1, a microsymbiont of Medicago laciniata (L.) miller collected in Lanzarote, Canary Islands, Spain
}

Wan Adnawani Meor Osman', Peter van Berkum², Milagros León-Barrios ${ }^{3}$, Encarna Velázquez ${ }^{4}$, Patrick Elia², Rui Tian ${ }^{1}$, Julie Ardley ${ }^{1}$ (D) Margaret Gollagher ${ }^{5}$, Rekha Seshadri ${ }^{6}$, T. B. K. Reddy ${ }^{6}$, Natalia Ivanova ${ }^{6}$, Tanja Woyke ${ }^{6}$, Amrita Pati', Victor Markowitz', Mohamed N. Baeshen ${ }^{8}$, Naseebh Nabeeh Baeshen ${ }^{8}$, Nikos Kyrpides ${ }^{6}$ and Wayne Reeve ${ }^{1 *}$

\begin{abstract}
Ensifer meliloti Mlalz-1 (INSDC = ATZD00000000) is an aerobic, motile, Gram-negative, non-spore-forming rod that was isolated from an effective nitrogen-fixing nodule of Medicago laciniata (L.) Miller from a soil sample collected near the town of Guatiza on the island of Lanzarote, the Canary Islands, Spain. This strain nodulates and forms an effective symbiosis with the highly specific host M. laciniata. This rhizobial genome was sequenced as part of the DOE Joint Genome Institute 2010 Genomic Encyclopedia for Bacteria and Archaea-Root Nodule Bacteria (GEBA-RNB) sequencing project. Here the features of E. meliloti Mlalz-1 are described, together with high-quality permanent draft genome sequence information and annotation. The 6,664,116 bp high-quality draft genome is arranged in 99 scaffolds of 100 contigs, containing 6314 protein-coding genes and 74 RNA-only encoding genes. Strain Mlalz-1 is closely related to Ensifer meliloti IAM 12611 ${ }^{\top}$, Ensifer medicae A $321^{\top}$ and Ensifer numidicus ORS $1407^{\top}$, based on $16 S$ rRNA gene sequences. gANI values of $\geq 98.1 \%$ support the classification of strain Mlalz-1 as E. meliloti. Nodulation of M. laciniata requires a specific nodC allele, and the nodC gene of strain Mlalz- 1 shares $\geq 98 \%$ sequence identity with nodC of M. laciniata-nodulating Ensifer strains, but $\leq 93 \%$ with nodC of Ensifer strains that nodulate other Medicago species. Strain Mlalz-1 is unique among sequenced E. meliloti strains in possessing genes encoding components of a T2SS and in having two versions of the adaptive acid tolerance response IpiA-acvB operon. In E. medicae strain WSM419, IpiA is essential for enhancing survival in lethal acid conditions. The second copy of the IpiA-acvB operon of strain Mlalz-1 has highest sequence identity (> 96\%) with that of $E$. medicae strains, which suggests genetic recombination between strain Mlalz-1 and E. medicae and the horizontal gene transfer of IpiA-acvB.
\end{abstract}

Keywords: Root-nodule bacteria, Ensifer, Geba-Rnb, Medicago, IpiA-acvB operon

\section{Introduction}

Symbiotic nitrogen fixation by pasture legumes and their associated root nodule bacteria provides a critical contribution to sustainable animal and plant production, and the maintenance of soil fertility in agricultural systems [1-3]. As such, it is of direct relevance to maintaining environmentally sustainable high agricultural yields,

\footnotetext{
* Correspondence: W.Reeve@murdoch.edu.au

${ }^{1}$ School of Veterinary and Life Sciences, Murdoch University, Murdoch, WA, Australia

Full list of author information is available at the end of the article
}

which significantly contributes to the Sustainable Development Goals adopted in September 2015 as part of the UN's development agenda 'Transforming our world: the 2030 Agenda for Sustainable Development' [4]. Medics (Medicago spp.) are some of the most important and extensively grown pasture legumes and their specific symbiosis with strains of rhizobia belonging to either Ensifer (synonym Sinorhizobium) meliloti or the closely related species $E$. medicae $[5,6]$ has been the subject of extensive research efforts [7]. 
Medicago laciniata (L.) Miller (cut leaf medic), an annual native of southern and eastern Mediterranean and Saharo-Sindian countries, is of importance because of its ability to grow in comparatively arid habitats and marginal cropping areas [8-11]. It is highly specific in its rhizobial requirements, forming a symbiosis only with a restricted subset of $E$. meliloti and not with strains that nodulate Medicago sativa L. (alfalfa) or Medicago truncatula Gaertn. [12, 13]. This symbiotic specificity has been linked to the rhizobial nod genes, in particular a specific nodC allele [14]. For example, van Berkum and colleagues found that most rhizobial strains isolated from Tunisian $M$. truncatula and M. laciniata shared chromosomal identity, but differed in their nodC alleles [15]. Based on these and other differing symbiotic traits, Villegas et al. [13] proposed two biovars within E. meliloti: bv. medicaginis for Ensifer strains that are symbiotically efficient on $M$. laciniata and bv. meliloti for the classical E. meliloti group that efficiently nodulates $M$. sativa. However, in subsequent studies the diversity observed within bv. medicaginis strains indicate that this group is certainly heterogeneous [16].

$M$. laciniata is native to the Canary Islands and is present on all of the islands of this archipelago, growing in environments that range from arid to subhumid. Ensifer meliloti strain Mlalz-1 was isolated from a $\mathrm{N}_{2}$-fixing nodule of $M$. laciniata grown in alkaline soil $(\mathrm{pH} 9.0)$ collected in Guatiza, in the arid Northeast of Lanzarote Island, in 2007. This strain was one of the rhizobial genomes sequenced as part of the DOE Joint Genome Institute 2010 GEBA-RNB project proposal [17, 18]. Here an analysis of the complete genome sequence of $E$. meliloti Mlalz-1 is provided.

\section{Organism information}

\section{Classification and features}

E. meliloti Mlalz-1 is a motile, non-sporulating, nonencapsulated, Gram-negative strain in the class Alphaproteobacteria. The rod shaped form has dimensions of approximately $0.5 \mu \mathrm{m}$ in width and $1.0-2.0 \mu \mathrm{m}$ in length (Fig. 1 Left and Center). It is fast growing, forming colonies after 3-5 days when grown on $1 / 2 \mathrm{LA}$, TY, or a modified yeast-mannitol agar [19] at $28{ }^{\circ} \mathrm{C}$. Colonies on $1 / 2 \mathrm{LA}$ are opaque, slightly domed and moderately mucoid with smooth margins (Fig. 1 Right). Minimum Information about the Genome Sequence (MIGS) for strain Mlalz-1 is provided in Table 1 and Additional file 1: Table S1.

\section{Symbiotaxonomy}

$M$. laciniata is a highly specific host and its microsymbionts also appear to be highly specific since studies of Medicago isolates have shown that $M$. laciniata strains fail to nodulate a range of Medicago species [5, 12]. Bailly et al. [20] reported that isolates of M. laciniata nodulated and fixed nitrogen with $M$. truncatula, but also provided evidence that these were the progeny of horizontal transfer of the nodulation genes. Strain Mlalz-1 nodulates and is effective for nitrogen fixation with $M$. laciniata. We report here that strain Mlalz-1 is unable to nodulate Medicago polymorpha L., the definitive host for E. medicae strains [6].

\section{Extended feature descriptions}

Previous studies using multilocus sequence typing showed that M. laciniata rhizobia did not form a distinct chromosomal group [15]. Phylogenetic analysis of strain Mlalz-1 was performed by aligning the 16S rRNA sequence (1389 bp from scaffold 84.85 ) to the 16S rRNA gene sequences of Ensifer type strains (Fig. 2). Based on four variable sites within this $16 \mathrm{~S}$ rRNA gene sequence alignment, strain Mlalz-1 is closely related to E. meliloti IAM $12611^{\mathrm{T}}\left(=\right.$ LMG $\left.6133^{\mathrm{T}}\right)$ [21], E. medicae A $321^{\mathrm{T}}(=$ LMG 19920 ${ }^{\mathrm{T}}$ ) [6] and E. numidicus ORS $1407^{\mathrm{T}}$ [22]. The available IMG 16S rRNA sequence of strain Mlalz-1 gave alignment identities of $100 \%$ to E. meliloti IAM $12611^{\mathrm{T}}, 99.7 \%$ to $E$. medicae A $321^{\mathrm{T}}$ and $99.5 \%$ to $E$. numidicus ORS $1407^{\mathrm{T}}$. In contrast, E. meliloti IAM
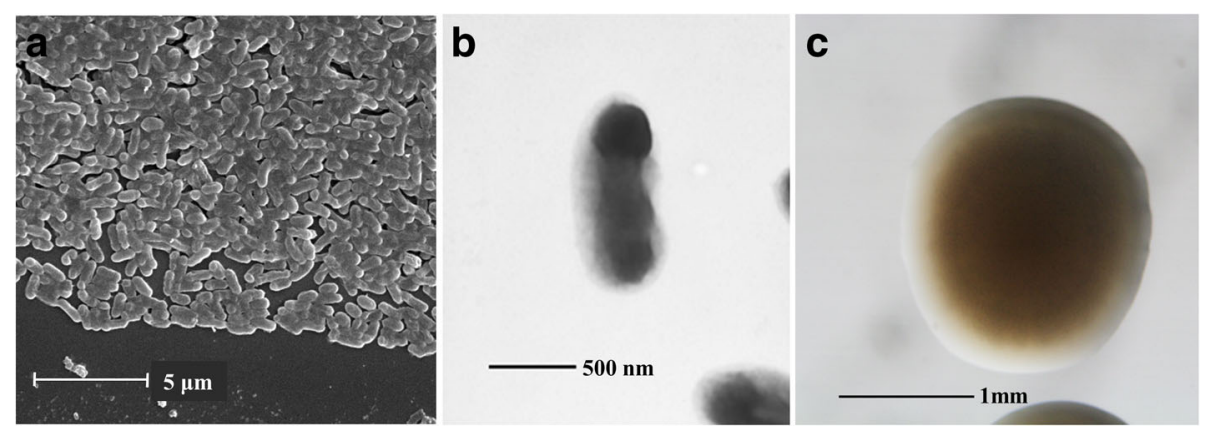

Fig. 1 Images of Ensifer meliloti Mlalz-1 using scanning (Left (a)) and transmission (Center (b)) electron microscopy as well as light microscopy to visualize colony morphology on solid media (Right (c)) 
Table 1 Classification and general features of Ensifer meliloti Mlalz-1 in accordance with the MIGS recommendations [65] published by the Genomic Standards Consortium [66]

\begin{tabular}{|c|c|c|c|}
\hline MIGS ID & Property & Term & Evidence code $^{a}$ \\
\hline & Current classification & Domain Bacteria & TAS [67] \\
\hline & & Phylum Proteobacteria & TAS [68] \\
\hline & & Class Alphaproteobacteria & $\operatorname{TAS}[69,70]$ \\
\hline & & Order Rhizobiales & $\operatorname{TAS}[70,71]$ \\
\hline & & Family Rhizobiaceae & $\operatorname{TAS}[72,73]$ \\
\hline & & Genus Ensifer & TAS [74] \\
\hline & & Species Ensifer meliloti & {$[21]$} \\
\hline & & Strain: Mlalz-1 (= USDA 1984) & IDA \\
\hline & Gram stain & Negative & IDA \\
\hline & Cell shape & Rod & IDA \\
\hline & Motility & Motile & IDA \\
\hline & Sporulation & Non-sporulating & NAS \\
\hline & Temperature range & $10-40^{\circ} \mathrm{C}$ & IDA \\
\hline & Optimum temperature & $25-30^{\circ} \mathrm{C}$ & IDA \\
\hline & pH range; Optimum & $5-9.5 ; 6.5-8$ & IDA \\
\hline & Carbon source & Varied & IDA \\
\hline MIGS-6 & Habitat & Soil; root nodule on host Medicago laciniata (L.) Miller & IDA \\
\hline MIGS-6.3 & Salinity & Tolerates 0 to $1 \%(w / v) \% \mathrm{NaCl}$ & TAS \\
\hline MIGS-22 & Oxygen requirement & Aerobic & IDA \\
\hline MIGS-15 & Biotic relationship & Free living, symbiotic & IDA \\
\hline MIGS-14 & Pathogenicity & Biosafety level 1 & TAS [75] \\
\hline MIGS-4 & Geographic location & Guatiza, Lanzarote, Canary Islands, Spain & IDA \\
\hline MIGS-5 & Sample collection date & 2007 & IDA \\
\hline MIGS-4.1 & Latitude & 29.074324 & IDA \\
\hline MIGS-4.2 & Longitude & -13.479696 & IDA \\
\hline MIGS-4.3 & Depth & $5-10 \mathrm{~cm}$ & IDA \\
\hline MIGS-4.4 & Altitude & $102 \mathrm{~m}$ & IDA \\
\hline
\end{tabular}

avidence codes - IDA Inferred from Direct Assay, TAS Traceable Author Statement (i.e., a direct report exists in the literature), NAS Non-traceable Author Statement (i.e., not directly observed for the living, isolated sample, but based on a generally accepted property for the species, or anecdotal evidence). Evidence codes are from the Gene Ontology project $[76,77]$

$12611^{\mathrm{T}}$ and Ensifer terangae LMG $7834^{\mathrm{T}}$ [23] were only 97.3\% similar.

\section{Genome sequencing information}

\section{Genome project history}

E. meliloti Mlalz-1 was selected for sequencing at the U.S. Department of Energy funded Joint Genome Institute as part of the GEBA-RNB project $[17,18]$. The root nodule bacteria in this project were selected based on environmental and agricultural relevance to issues in global carbon cycling, alternative energy production, and biogeochemical importance. In particular, strain Mlalz-1 was chosen since it has strict host specificity for $M$. laciniata, which is suited for cultivation in arid environments [11]. The E. meliloti Mlalz-1 genome project is deposited in the Genomes Online Database [24] and a high-quality permanent draft genome sequence (IMG Genome ID 2513237143) is deposited in IMG [25]. Sequencing, finishing and annotation were performed by the JGI. A summary of the project information is shown in Table 2.

\section{Growth conditions and genomic DNA preparation}

E. meliloti Mlalz-1 (= USDA 1984) was cultured on MAG solid media [26] for three days at $28{ }^{\circ} \mathrm{C}$ to obtain well grown, well separated colonies, then a single colony was selected from the plate and inoculated into $5 \mathrm{ml}$ MAG broth media. The culture was grown for $48 \mathrm{~h}$ on a gyratory shaker $(200 \mathrm{rpm})$ at $28{ }^{\circ} \mathrm{C}$. Subsequently $1 \mathrm{ml}$ was used to inoculate $50 \mathrm{ml}$ of MAG and the cells were 


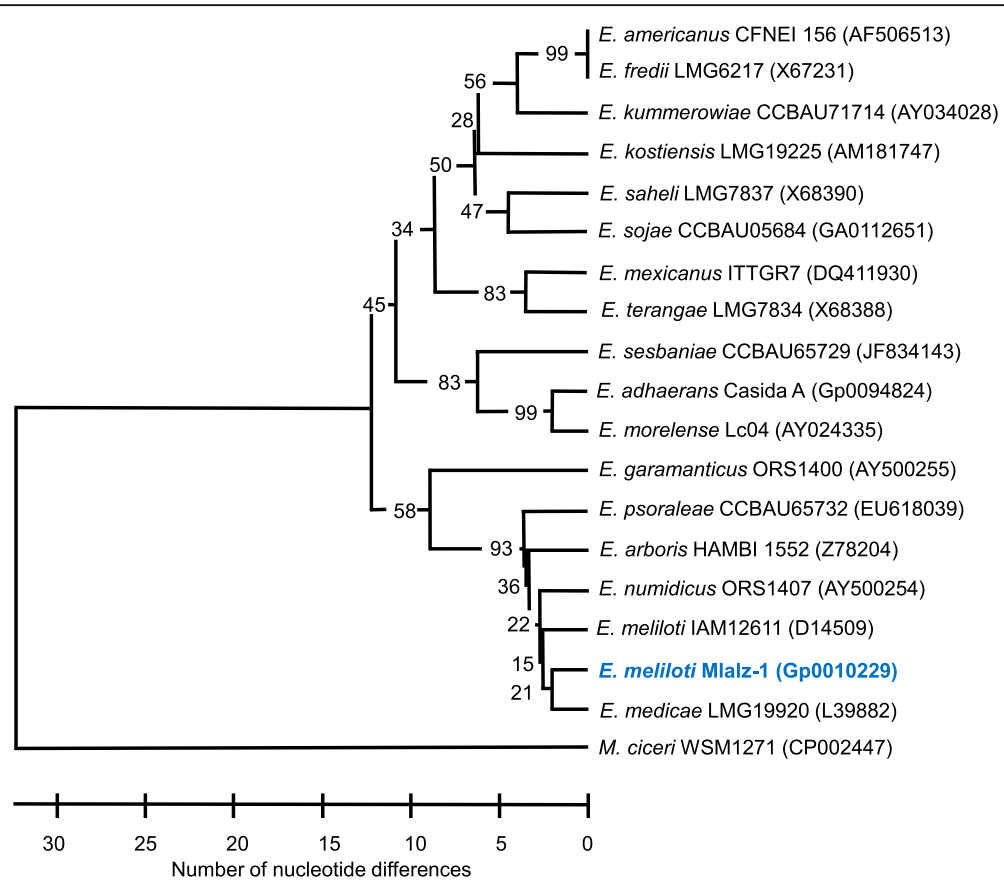

Fig. 2 Comparison of the $16 \mathrm{~S}$ rRNA gene sequences of Ensifer meliloti Mlalz-1 (shown in bold) and other Ensifer spp. type strains, based on aligned $16 \mathrm{~S}$ rRNA gene sequences of $1389 \mathrm{bp}$. Of the 1389 sites, 1279 were constant and 48 were informative. There were eight gaps overall when Mesorhizobium ciceri bv biserrulae WSM1271 was included in the analysis. Phylogenetic analysis was done using MEGA, version 6.0 [61] after manually assembling the alignment by using GeneDoc version 2.6.001 [62]. M. ciceri bv biserrulae WSM1271 was used as an outgroup and the tree was assembled using the UPGMA algorithm based on the number of nucleotide differences. This approach was used since the potential for genetic recombination among the different 165 rRNA genes as reported by van Berkum [63] cannot be ignored. Bootstrap analysis [64] with 2000 permutations of the data set was done to assess support for the branch points. Strains with a genome sequencing project registered in GOLD [24] are Ensifer adhaerens Casida $A^{\top}$, M. ciceri bv. biserrulae WSM1271 and Mlalz-1 and the GOLD ID is provided in place of the GenBank accession number

Table 2 Genome sequencing project information for Ensifer meliloti Mlalz-1

\begin{tabular}{lll}
\hline MIGS ID & Property & Term \\
\hline MIGS-31 & Finishing quality & High-quality permanent draft \\
MIGS-28 & Libraries used & Illumina Standard PE \\
MIGS-29 & Sequencing platforms & Illumina HiSeq 2000 \\
MIGS-31.2 & Fold coverage & $748 \times$ \\
MIGS-30 & Assemblers & Velvet version 1.1.04; \\
& & ALLPATHS V. r39750 \\
MIGS-32 & Gene calling methods & Prodigal 1.4 \\
& Locus Tag & A3CA [78] \\
& GenBank ID & ATZD000000000 \\
& Genbank Date of Release & January 30 2012 \\
& GOLD ID & Gp0010229 [79] \\
& BIOPROJECT & 165,343 \\
MIGS-13 & Source Material Identifier & Mlalz-1 (=USDA 1984) \\
& Project relevance & Symbiotic N fixation, $^{2}$ \\
& & agriculture \\
\hline
\end{tabular}

incubated on a gyratory shaker $(200 \mathrm{rpm})$ at $28{ }^{\circ} \mathrm{C}$ until an $\mathrm{OD}_{600 \mathrm{~nm}}$ of 0.6 was reached. DNA was isolated from $50 \mathrm{ml}$ of cells by Peter van Berkum according to the method described by van Berkum [26]. The final concentration of the DNA was set to $0.5 \mathrm{mg} \mathrm{ml}^{-1}$.

\section{Genome sequencing and assembly}

The draft genome of E. meliloti Mlalz-1 was generated at the DOE Joint genome Institute (JGI) using Illumina technology [27]. An Illumina standard PE library was constructed and sequenced using the Illumina HiSeq 2000 platform that generated 35,720,836 reads totalling $4983 \mathrm{Mbp}$. All general aspects of library construction and sequencing were done at the JGI and details can be found on the JGI website [28]. All raw Illumina sequence data was passed through DUK, a filtering program developed at JGI, which removes known Illumina sequencing and library preparation artefacts (Mingkun L, Copeland A, Han J; unpublished). The following steps for assembly were: (1) filtered Illumina reads were assembled using Velvet (version 1.1.04) [29]; (2) 1-3 Kbp simulated paired end reads were created from Velvet contigs using wgsim (version 0.3.0) [30]; (3) Illumina reads were 
assembled with simulated read pairs using Allpaths-LG (version r39750) [31]. Parameters for the assembly steps were 1) Velvet: -v -s 51 -e 71 -i 2 -t 1 -f "-shortPaired -fastq \$FASTQ" -o "-ins_length 250 -min_contig_lgth 500" for Velvet and 2) wgsim: -e 0-1 76-2 76 -r 0 -R 0 -X 0. The final draft assembly contained 100 contigs in 99 scaffolds. The total size of the genome is $6.7 \mathrm{Mbp}$ and the final assembly is based on $4983 \mathrm{Mbp}$ of Illumina data, which provides an average of $748 \times$ coverage of the genome.

\section{Genome annotation}

Genes were identified using Prodigal [32], as part of the DOE-JGI genome annotation pipeline [33, 34]. The predicted CDSs were translated and used to search the National Center for Biotechnology Information nonredundant database, UniProt, TIGRFam, Pfam, KEGG, COG, and InterPro databases. The tRNAScanSE tool [35] was used to find tRNA genes, whereas ribosomal RNA genes were found by searches against models of the ribosomal RNA genes built from SILVA [36]. Other non-coding RNAs such as the RNA components of the protein secretion complex and the RNase $\mathrm{P}$ were identified by searching the genome for the corresponding Rfam profiles using INFERNAL [37]. Additional gene prediction analysis and manual functional annotation was done within the Integrated Microbial GenomesExpert Review platform [38] developed by the Joint Genome Institute, Walnut Creek, CA, USA.

\section{Genome properties}

The genome is $6,664,116$ bp with $62.16 \%$ GC content (Table 3) and comprised of 99 scaffolds. From a total of 6388 genes, 6314 were protein encoding and 74 RNA

Table 3 Genome statistics for Ensifer meliloti Mlalz-1

\begin{tabular}{lll}
\hline Attribute & Value & \% of Total \\
\hline Genome size (bp) & $6,664,116$ & 100.00 \\
DNA coding (bp) & $5,754,332$ & 86.35 \\
DNA G + C (bp) & $4,142,407$ & 62.16 \\
DNA scaffolds & 99 & 100.00 \\
Total genes & 6388 & 100.00 \\
Protein-coding genes & 6314 & 98.84 \\
RNA genes & 74 & 1.16 \\
Pseudo genes & 0 & 0.00 \\
Genes in internal clusters & 1054 & 16.50 \\
Genes with function prediction & 5080 & 79.52 \\
Genes assigned to COGs & 4659 & 72.93 \\
Genes with Pfam domains & 5317 & 83.23 \\
Genes with signal peptides & 555 & 8.69 \\
Genes with transmembrane helices & 1440 & 22.54 \\
CRISPR repeats & 0 & 0.00 \\
\hline
\end{tabular}

only encoding genes. Most genes (79.52\%) were assigned a putative function whilst the remaining genes were annotated as hypothetical. The distribution of genes into COGs functional categories is presented in Table 4.

\section{Insights from the genome sequence}

E. meliloti Mlalz-1 is one of seven strains of E. meliloti that have been sequenced from the GEBA-RNB genome sequencing projects [17]. On the basis of $16 \mathrm{~S}$ rRNA sequence identity, strain Mlalz-1 is closely related to $E$. meliloti IAM $12611^{\mathrm{T}}\left(=\right.$ LMG $\left.6133^{\mathrm{T}}\right)$, E. medicae A $321^{\mathrm{T}}\left(=\right.$ LMG $\left.19920^{\mathrm{T}}\right)$ and E. numidicus ORS $1407^{\mathrm{T}}$. As the genomes of these type strains have not been sequenced or are not publically available, gANI values [39]

Table 4 Number of genes of Ensifer meliloti Mlalz-1 associated with the general COG functional categories

\begin{tabular}{|c|c|c|c|}
\hline Code & Value & \%age & Description \\
\hline$J$ & 217 & 4.09 & $\begin{array}{l}\text { Translation, ribosomal structure and } \\
\text { biogenesis }\end{array}$ \\
\hline A & 0 & 0.00 & RNA processing and modification \\
\hline K & 466 & 8.77 & Transcription \\
\hline$L$ & 122 & 2.3 & Replication, recombination and repair \\
\hline B & 1 & 0.02 & Chromatin structure and dynamics \\
\hline D & 39 & 0.73 & $\begin{array}{l}\text { Cell cycle control, cell division, } \\
\text { chromosome partitioning }\end{array}$ \\
\hline Y & 0 & 0.00 & Nuclear structure \\
\hline V & 117 & 2.20 & Defense mechanisms \\
\hline $\mathrm{T}$ & 216 & 4.07 & Signal transduction mechanisms \\
\hline M & 301 & 5.67 & $\begin{array}{l}\text { Cell wall/membrane/envelope } \\
\text { biogenesis }\end{array}$ \\
\hline $\mathrm{N}$ & 72 & 1.36 & Cell motility \\
\hline Z & 0 & 0.00 & Cytoskeleton \\
\hline W & 33 & 0.62 & Extracellular structures \\
\hline$U$ & 74 & 1.39 & $\begin{array}{l}\text { Intracellular trafficking, secretion, } \\
\text { and vesicular transport }\end{array}$ \\
\hline $\mathrm{O}$ & 206 & 3.88 & $\begin{array}{l}\text { Posttranslational modification, protein } \\
\text { turnover, chaperones }\end{array}$ \\
\hline C & 358 & 6.74 & Energy production and conversion \\
\hline G & 555 & 10.45 & Carbohydrate transport and metabolism \\
\hline$E$ & 584 & 10.99 & Amino acid transport and metabolism \\
\hline $\mathrm{F}$ & 116 & 2.18 & Nucleotide transport and metabolism \\
\hline $\mathrm{H}$ & 242 & 4.56 & Coenzyme transport and metabolism \\
\hline I & 220 & 4.14 & Lipid transport and metabolism \\
\hline$P$ & 279 & 5.25 & Inorganic ion transport and metabolism \\
\hline Q & 159 & 2.99 & $\begin{array}{l}\text { Secondary metabolite biosynthesis, } \\
\text { transport and catabolism }\end{array}$ \\
\hline $\mathrm{R}$ & 551 & 10.37 & General function prediction only \\
\hline S & 348 & 6.55 & Function unknown \\
\hline$x$ & 36 & 0.68 & Mobilome: prophages, transposons \\
\hline- & 1729 & 27.07 & Not in COGS \\
\hline
\end{tabular}


had to be compared with other fully sequenced Ensifer strains (Table 5). E. meliloti Mlalz-1 currently forms a gANI clique with other E. meliloti strains (gANI values $\geq 98.14 \%$ ), compared with gANI values of $\leq 87.9 \%$ with the finished genomes of other Ensifer strains. This supports the classification of strain Mlalz-1 as an E. meliloti strain, in accordance with the defined species affiliation cut-off value of $96.5 \%$ gANI [39]. The total genome size of strain Mlalz-1 is $6.6 \mathrm{Mbp}$, which falls within the expected size range of 6.6-8.9 Mbp for E. meliloti. The genome architecture of $E$. meliloti consists of a chromosome and the two symbiotic megaplasmids pSymA and pSymB [20]. Replication of a plasmid is initiated by the replication protein encoded by $r e p C$, which is present as a single copy on E. meliloti $\mathrm{pSymA}$ and $\mathrm{pSymB}$. The $E$. meliloti Mlalz-1 genome carried 2 repC loci (A3CADRAFT 00120 and A3CADRAFT 01676) with highest encoded protein identity to RepC proteins of $E$. meliloti strains. Mlalz-1 A3CADRAFT_00120 RepC1 had highest identity $(98.10 \%)$ to the RepC1 protein encoded by SMb20044 on pSymB of E. meliloti 1021. E. meliloti Mlalz-1 A3CADRAFT_01676 RepC2 had highest identity $(99.00 \%)$ to the RepC2 protein encoded by SMa2391 on pSymA of E. meliloti 1021. This indicated the presence of two megaplasmids in strain Mlalz-1, and revealed that strain Mlalz-1 has a similar genome architecture to that of E. meliloti 1021.

\section{Extended insights}

All 29 E. meliloti strains within the gANI clique share a core set of 4948 orthologous genes, using cut off values of $1 \mathrm{e}-5$ and $30 \%$ minimum protein identity. E. meliloti Mlalz-1 contains 176 unique genes, 96 (54.5\%) of which encode hypothetical proteins. The unique genes include those encoding the components of a T2SS, located on scaffold A3CADRAFT_scaffold_5.6 (Fig. 3a), as well as genes that encode a DNA methyltransferase and a NitT/ TauT family transport system. These T2SS components form part of a unique COG profile generated for Mlaz-1 (Table 6). The T2SS secretion system is used to translocate a wide range of proteins from the periplasm across the outer membrane [40]. Although T2SS genes are not found in other E. meliloti strains or in the Ensifer fredii strains GR64 and USDA 257, they are present in the genomes of the E. fredii strains HH103 and NGR234, in a similar gene arrangement to that observed in E. meliloti Mlalz-1 [41, 42] (Fig. 3b). Generally, the T2SS gene cluster is comprised of $12-15$ genes, and strain Mlalz-1 contains the 12 required genes gspDOGLMCKEFHIJ necessary for a functional T2SS, but lacks the gspS gene found only in certain genera [43] (Fig. 3c).

In common with some other E. meliloti strains, strain Mlalz-1 contains several genes encoding phage components. The PHASTER algorithm [44] was used to identify two resident prophages, present on scaffold A3CADRAFT_scaffold_4.5: one that was incomplete (Prophage Region 1) and one that was intact (Prophage Region 2) (Fig. 4). The proteins encoded by Prophage Region $1(11.4 \mathrm{~kb})$ and Prophage Region $2(55 \mathrm{~kb})$ were most closely related to the phage proteins of PHAGE_Mycoba_Catalina_NC031238 and PHAGE_Sinorh_phiLM21_ NC_029046, respectively.

The Mlaz-1 genome also contains acid-tolerance or acid-responsive genes that are orthologous to the genes identified in the comparatively acid tolerant strain $E$. medicae WSM419. Acid-tolerance or acid-responsive genes identified in Mlaz-1 include actA $(\ln t), a c t P, a c t R$, actS, phrR, exoR, exoH, lpiA, acvB, degP1, mdh3, fbaB, groS, $k d p B, k d p C$, fixN2 and fixO2 [45-52] (Additional file 2: Table S2). It is notable that strain Mlalz-1 is unique among the sequenced Ensifer strains since it contains two versions of the highly acid-induced lpiA-acvB operon. One operon (A3CADRAFT_01189A3CADRAFT_01190) is found on scaffold A3CADRAFT_scaffold_3.4, in a gene region that is conserved in other $E$. meliloti (sequence similarity $>98 \%$ ) and is located on the

Table 5 Pairwise gANI comparisons of selected finished genomes of sequenced Ensifer strains

\begin{tabular}{|c|c|c|c|c|c|c|c|c|c|c|c|}
\hline Strain & Gold ID: Gp & Casida A & USDA 257 & WSM 419 & 1021 & AK83 & BL225C & GR4 & Mlalz-1 & Rm41 & SM11 \\
\hline E.adhaerens Casida A & 0094824 & 100 & 80.5 & 79.06 & 80.12 & 80.11 & 80.06 & 80.01 & 80.08 & 80.03 & 80.06 \\
\hline E. fredii USDA 257 & 0005169 & 80.5 & 100 & 81.89 & 83.26 & 83.24 & 83.25 & 83.20 & 83.14 & 83.33 & 83.22 \\
\hline E. medicae WSM419 & 0000117 & 79.06 & 81.93 & 100 & 88.18 & 88.13 & 88.26 & 88.24 & 87.90 & 88.14 & 88.26 \\
\hline E. meliloti 1021 & 0000726 & 80.12 & 83.26 & 88.19 & 100 & 99.36 & 99.62 & 99.41 & 98.80 & 99.24 & 99.43 \\
\hline E. meliloti AK83 & 0006695 & 80.08 & 83.25 & 88.16 & 99.36 & 100 & 99.33 & 99.14 & 98.60 & 99.38 & 99.33 \\
\hline E. meliloti BL225C & 0006560 & 80.06 & 83.25 & 88.28 & 99.62 & 99.33 & 100 & 99.44 & 98.81 & 99.26 & 99.39 \\
\hline E. meliloti GR4 & 0020501 & 80.01 & 83.23 & 88.26 & 99.41 & 99.14 & 99.43 & 100 & 98.81 & 99.05 & 99.25 \\
\hline E. meliloti Mlalz-1 & 0010229 & 80.11 & 83.15 & 87.91 & 98.80 & 98.59 & 99.81 & 98.81 & 100 & 98.59 & 98.66 \\
\hline E. meliloti Rm41 & 0025853 & 80.05 & 83.36 & 88.11 & 99.26 & 99.39 & 99.25 & 99.06 & 98.59 & 100 & 99.33 \\
\hline E. meliloti SM11 & 0006018 & 80.05 & 83.23 & 88.29 & 99.45 & 99.33 & 99.39 & 99.26 & 98.67 & 99.32 & 100 \\
\hline
\end{tabular}

For E. meliloti Mlalz-1, gANI values above the microbial species delineation cutoff value of $96.5 \%$ [39] are in bold font 
a Emel Mlalz-1: A3CADRAFT_scaffold_5.6
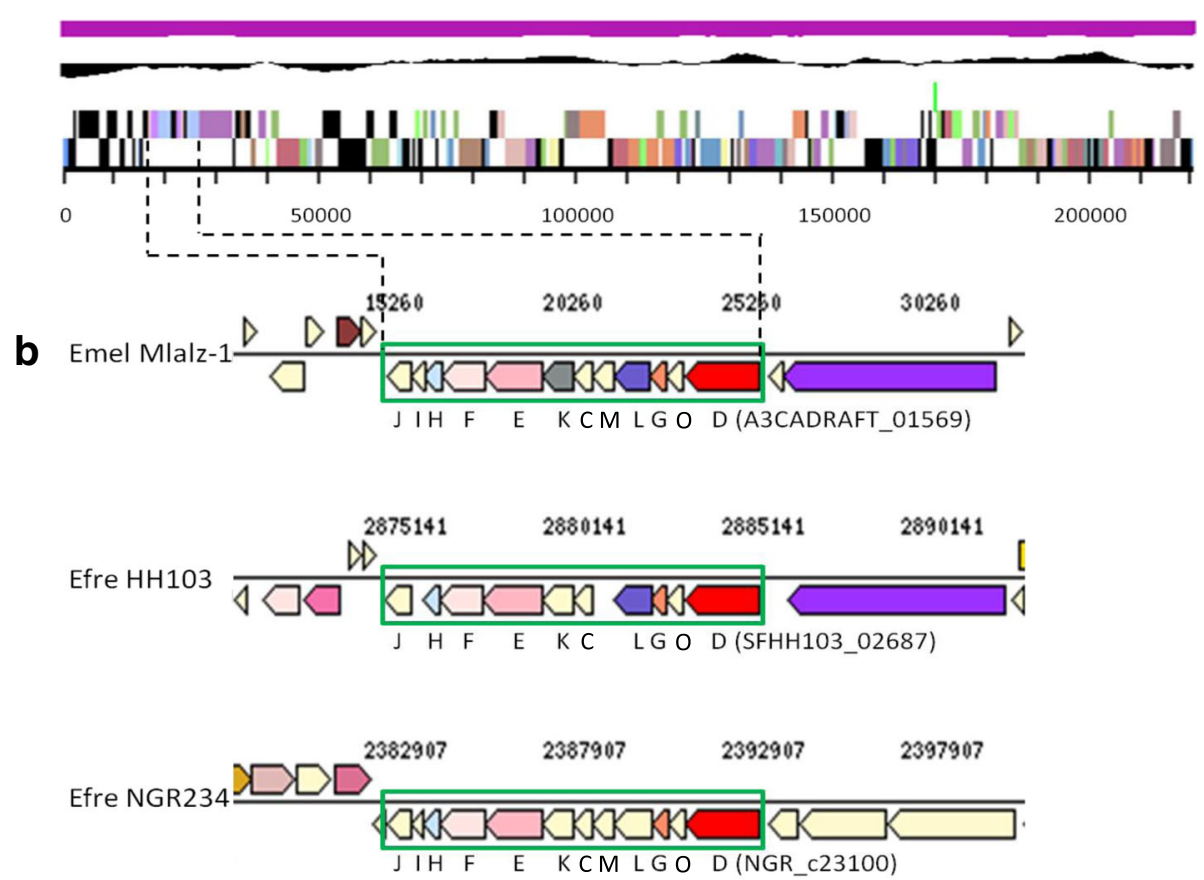

C

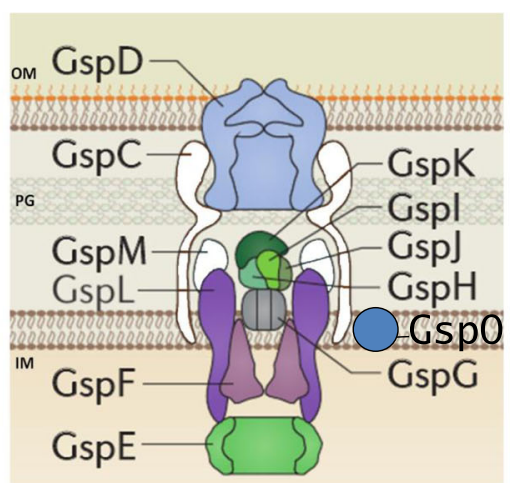

Fig. 3 a Map of Ensifer meliloti Mlalz-1: A3CADRAFT_scaffold_ 5.6. b Genetic organization of the T2SS clusters in E. meliloti Mlalz-1 (Emel Mlalz-1), Ensifer fredii $\mathrm{HH} 103$ (Efre HH103) and Ensifer fredii NGR234 (Efre NGR234). J, gspj; l, gspl; H, gspH; F, gspF; E, gspE; K, gspK; C, gspC; M, gspM; L, gspL; G, gspG; O, gspO; D, gspD. c Schematics of the T2SS of Gram-negative bacteria [43]. The secretin, GspD (A3CADRAFT_01569); the polytopic protein, GspF (A3CADRAFT_01561); the cytoplasmic ATPase, GspE (A3CADRAFT_01562); the major pseudopilin component, GspG (A3CADRAFT_01567); the minor pseudopilins, GspH (A3CADRAFT_01560), Gspl (A3CADRAFT_01559), GspJ (A3CADRAFT_01558) and GspK (A3CADRAFT_01563); the bitopic proteins, GspL (A3CADRAFT_01566), GspC (A3CADRAFT_01564) and GspM (A3CADRAFT_01565); the peptidase, GspO (A3CADRAFT_01568)(GspS is absent from Mlalz-1); OM, outer membrane; $P G$, peptidoglycan; IM, inner membrane

chromosome of the fully sequenced E. meliloti 1021 . The second version of the lpiA-acvB operon (A3CADRAFT_05694-A3CADRAFT_05695) is located on A3CADRAFT_scaffold_47.48, in a gene region that is conserved in E. medicae genomes (sequence similarity $>96 \%$ ) and is located on the pSMED02 symbiotic plasmid of the fully sequenced $E$. medicae WSM419. The regulatory gene $f s r R$, required for the acid activated expression of lpiA in E. medicae WSM419 [53], is located upstream of A3CADRAFT_05694 in strain Mlalz-1. This regulatory gene is absent from the
A3CADRAFT_01190 gene region, and from the lpiA-acvB gene regions of all other $E$. meliloti sequenced genomes. These findings suggest that $E$. meliloti Mlalz- 1 acquired the plasmid-borne lpiA-acvB operon and associated $f_{s} r R$ regulatory gene by lateral transfer from an $E$. medicae strain.

Essential symbiotic (nod, nif and fix) genes identified in the E. meliloti Mlalz-1 genome (Additional file 2: Table S3 and S4) are located in several clusters on the following scaffolds: A3CADRAFT_scaffold_54.55 (Fig. 5a), A3CA DRAFT_scaffold_61.62 (Fig. 5b), A3CADRAFT_scaffold_63.64 (Fig. 5c), A3CADRAFT_scaffold_71.72 (Fig. 5d) 
Table 6 List of the unique COGs in Ensifer meliloti Mlalz-1

\begin{tabular}{lllll}
\hline COG & Name & Locus Tag & Gene symbol & Protein function \\
\hline 0393 & Uncharacterized conserved protein YbjQ, UPF0145 family & A3CADRAFT_01446 & & Unknown \\
4970 & Tfp pilus assembly protein FimT & A3CADRAFT_01560 & gspH & T2SS \\
1459 & Type II secretory pathway, component PulF & A3CADRAFT_01561 & gspF & T2SS \\
2804 & $\begin{array}{l}\text { Type II secretory pathway ATPase GspE/PulE or T4P pilus } \\
\text { assembly pathway ATPase PilB }\end{array}$ & A3CADRAFT_01562 & gspE & T2SS \\
3156 & Type II secretory pathway, component PulK & A3CADRAFT_01563 & gspK & T2SS \\
3166 & Tfp pilus assembly protein PilN & A3CADRAFT_01566 & gspL & T2SS \\
2165 & Type II secretory pathway, pseudopilin PulG & A3CADRAFT_01567 & gspG & T2SS \\
1450 & Type II secretory pathway component GspD/PulD (secretin) & A3CADRAFT_01569 & gspD & T2SS \\
2189 & Adenine specific DNA methylase Mod & A3CADRAFT_02454 & yhdJ & DNA methyltransferase \\
4705 & Uncharacterized membrane-anchored protein & A3CADRAFT_05679 & & Membrane protein \\
4089 & Uncharacterized membrane protein & A3CADRAFT_05685 & & Membrane protein \\
2021 & Homoserine acetyltransferase & A3CADRAFT_06155 & & Homoserine acetyltransferase \\
\hline
\end{tabular}

and A3CADRAFT_scaffold_74.75 (Fig. 5e). Nodulation of M. laciniata has been shown to require a specific nodC allele [14]. The nodC gene of strain Mlalz-1 has highest sequence identity ( $\geq 98 \%$ ) with nodC of other M. laciniatanodulating Ensifer strains in the NCBI database, whereas there is a lower sequence identity $(\leq 93 \%)$ with nodC of Ensifer strains that nodulate other Medicago species. Nodulation of Medicago hosts requires Nod factors that are sulfated at the reducing terminus and acylated at the nonreducing terminus, with a polyunsaturated fatty acyl tail $[54,55]$. The NodH sulfotransferase, together with the NodP and NodQ sulfate-activating complex, are required for Nod factor sulfation [56, 57]. Activity of NodL results in O-acetylation of the Nod factor [58], while NodE and NodF produce the specific polyunsaturated fatty acyl tail $[55,59]$.
Strain Mlalz-1 would appear to be typical of Ensifer strains that nodulate Medicago species since the nodEF, nodL and nodHPQ genes that are required for these specific decorations of the Nod factor are present in the genome. E. meliloti Mlalz-1 also possesses the three nodD genes that mediate host-specific activation of nod $A B C$ in the symbiotic interactions of E. meliloti with Medicago [60].

\section{Conclusions}

E. meliloti Mlalz-1 is a rhizobial strain that is able to nodulate and fix nitrogen with the highly specific host M. laciniata. Although the $16 \mathrm{~S}$ rRNA gene sequence divergence was insufficient to differentiate strain Mlalz-1 from E. meliloti, E. medicae or E. numidicus, a gANI value of $98.8 \%$ with the genome of E. meliloti 1021,

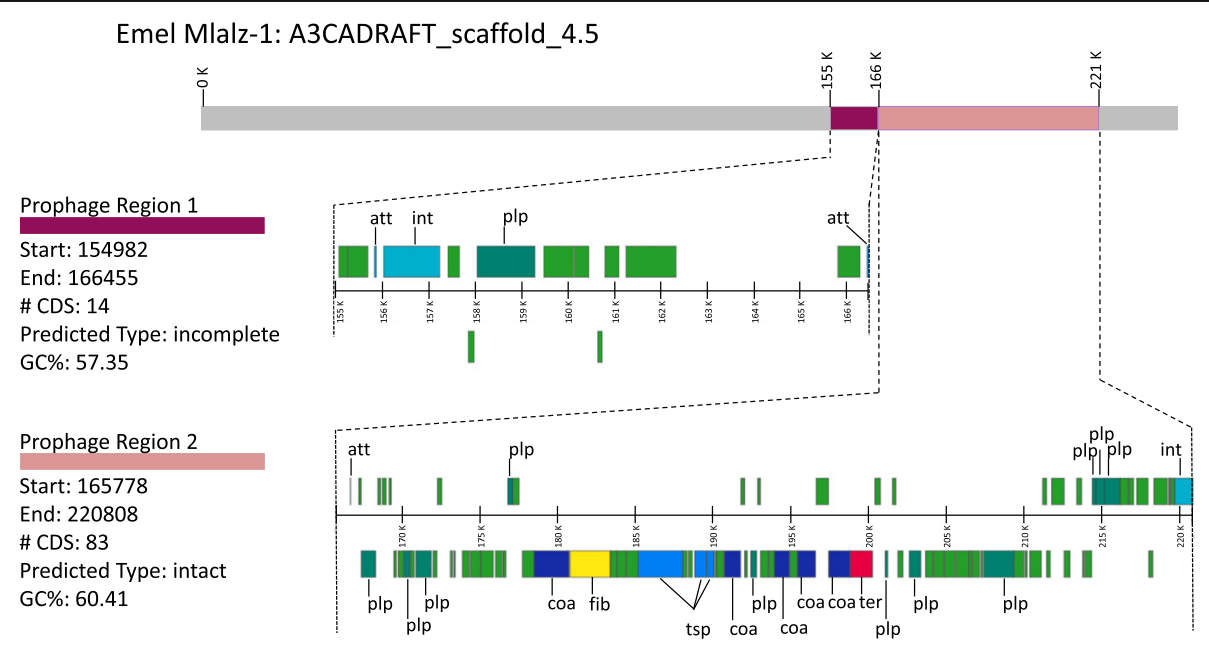

Fig. 4 Resident prophages present in Ensifer meliloti Mlalz-1 A3CADRAFT_scaffold_4.5, imaged using PHASTER [44]. Reference locus tag for Prophage Region 1 is A3CADRAFT_01439 (phage capsid family); reference locus tag for Prophage Region 2 is A3CADRAFT_01472 (prophage tail length tape measure protein). Prophage maps not drawn to scale. Attachment site (att), coat protein (coa), fiber protein (fib), integrase (int), phage-like protein (plp), tail shaft protein (tsp), and terminase (ter). All other genes encode hypothetical proteins 
a Emel Mlalz-1: A3CADRAFT_scaffold_54.55

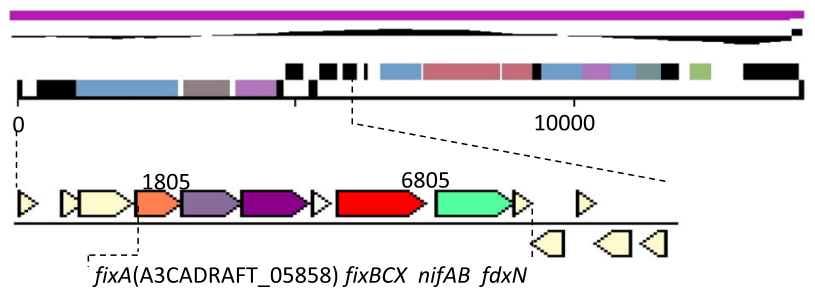

b Emel Mlalz-1: A3CADRAFT_scaffold_61.62

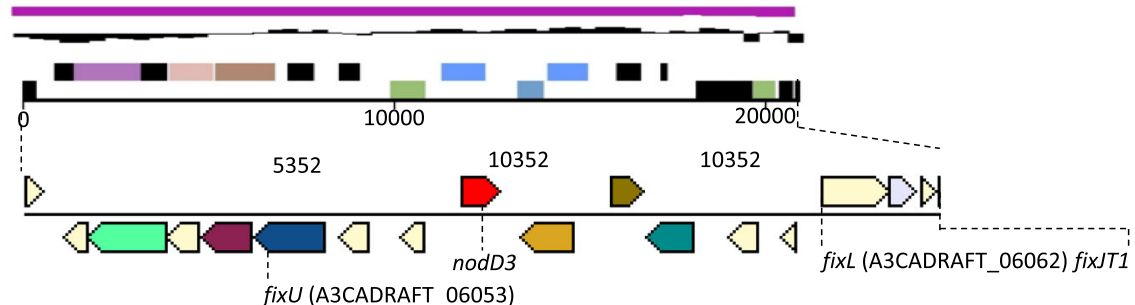

C Emel Mlalz-1: A3CADRAFT_scaffold_63.64

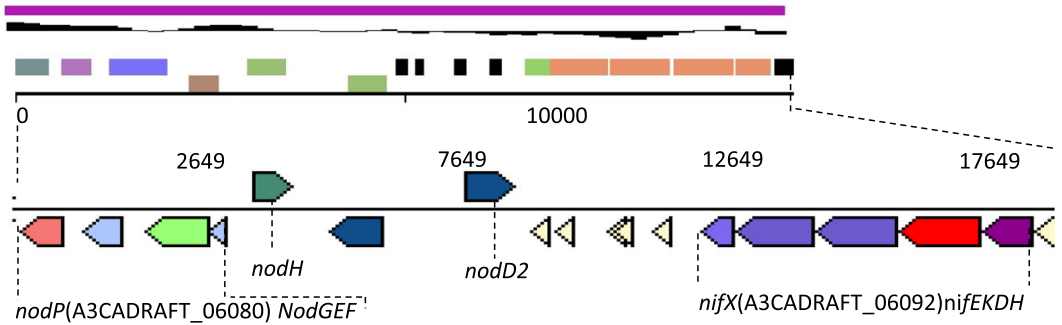

d Emel Mlalz-1: A3CADRAFT_scaffold_71.72

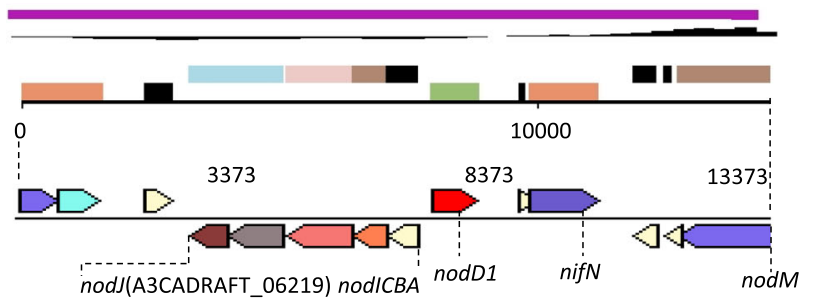

e Emel Mlalz-1: A3CADRAFT_scaffold_74.75

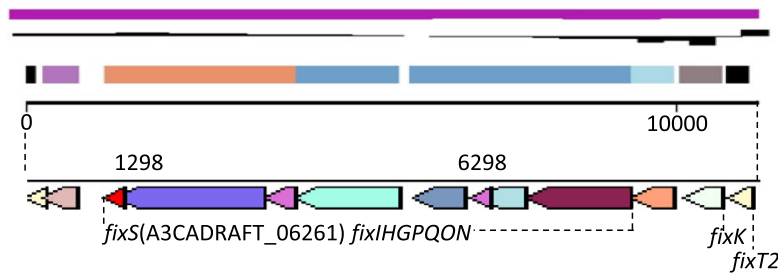

Fig. 5 Graphical map of the scaffolds; a A3CADRAFT_scaffold_54.55, b A3CADRAFT_scaffold_61.62, (c) A3CADRAFT_scaffold_63.64, d A3CADRAFT_scaffold_71.72 and e A3CADRAFT_scaffold_74.75 of Ensifer meliloti Mlalz-1 showing the location of common nodulation (nod) and fixation (nif and fix) genes within the symbiotic regions of this strain. From bottom to the top of the scaffold map: Genes on reverse strand (color by COG categories as denoted by the IMG platform), genes on forward strand (color by COG categories), RNA genes (tRNAs green, sRNAs red, other RNAs black), GC content, GC skew 
compared with $87.9 \%$ with the genome of $E$. medicae WSM419 identifies strain Mlalz-1 as E. meliloti. Nodulation of M. laciniata has been shown to be dependent on the presence of a specific nodC allele, which also is present in the genome of E. meliloti Mlalz-1, based on a $98 \%$ sequence identity with the nodC of other M. laciniata-nodulating Ensifer strains [14]. However, strain Mlalz-1 is unique among sequenced E. meliloti strains in possessing genes encoding components of a T2SS and in having two versions of the adaptive acid tolerance response lpiA-acvB operon. The second copy of the $E$. meliloti Mlalz-1 lpiA-acvB operon has highest sequence identity (>96\%) with that of sequenced E. medicae strains, which infers horizontal gene transfer of this region from $E$. medicae.

\section{Additional files}

Additional file 1: Table S1. Associated MIGS record for Ensifer meliloti Mlalz-1. (DOCX $52 \mathrm{~kb}$ )

Additional file 2: Table S2-S4. Table S2. Acid responsive gene orthologs present in Ensifer strains. Table S3. The nodulation genes of Ensifer meliloti Mlalz-1. Table S4. The nitrogen fixation genes of Ensifer meliloti Mlalz-1. (DOCX 65 kb)

\section{Abbreviations}

1/2LA: Half strength Lupin Agar; gANI: Genome-wide average nucleotide identity; GEBA-RNB: Genomic Encyclopedia for Bacteria and Archaea-Root Nodule Bacteria; IMG: Integrated Microbial Genomes; T2SS: Type II Secretion System; TY: Tryptone-yeast extract

\section{Acknowledgements}

We thank Gordon Thompson (Murdoch University) for the preparation of SEM and TEM photos. MLB thanks Alfredo Reyes-Betancort, from the Orotava Botanical Garden (Tenerife), for providing M. laciniata seeds.

\section{Funding}

This work was performed under the auspices of the US Department of Energy's Office of Science, Biological and Environmental Research Program, and by the University of California, Lawrence Berkeley National Laboratory under contract No. DE-AC02-05CH11231. We gratefully acknowledge the funding received from the Curtin University Sustainability Policy Institute and from Murdoch University's Small Research Grants Scheme in 2016.

\section{Authors' contributions}

MLB and EV isolated the strain and provided project metadata. PVB supplied the strain, the DNA and the background information for this project and participated in drafting the manuscript. PE curated the strain and performed sequence analysis of $16 \mathrm{~S}$ rRNA and nodC genes. RT supplied DNA to JGl and performed all imaging. JA provided symbiotic phenotype data. WAMO, JA and WR performed bioinformatics analyses and drafted the paper, $M B$ and NB provided financial support, and MG, RS, TBKR, NI, TW, AP, VM and NK were involved in sequencing the genome and/or editing the final paper. All authors read and approved the final manuscript.

\section{Competing interests}

The authors declare that they have no competing interests.

\section{Publisher's Note}

Springer Nature remains neutral with regard to jurisdictional claims in published maps and institutional affiliations.

\section{Author details}

${ }^{1}$ School of Veterinary and Life Sciences, Murdoch University, Murdoch, WA, Australia. ${ }^{2}$ U.S. Department of Agriculture, Soybean Genomics and
Improvement Laboratory, Beltsville Agricultural Research Center, 10300 Baltimore Avenue, Bldg. 006, Beltsville, MD 20705, USA. ${ }^{3}$ Departamento de Bioquímica, Microbiología, Biología Celular y Genética, Universidad de La Laguna, Tenerife, Spain. ${ }^{4}$ Departamento de Microbiología y Genetica and Instituto Hispanoluso de Investigaciones Agrarias (CIALE), Universidad de Salamanca, Salamanca, Spain. ${ }^{5}$ Curtin University Sustainability Policy Institute, Curtin University, Bentley, WA, Australia. ${ }^{6}$ DOE Joint Genome Institute, Walnut Creek, CA, USA. ${ }^{7}$ Biological Data Management and Technology Center, Lawrence Berkeley National Laboratory, Berkeley, CA, USA. ${ }^{8}$ Department of Biology, Faculty of Science, University of Jeddah, Jeddah, Saudi Arabia.

Received: 30 March 2017 Accepted: 14 September 2017

Published online: 25 September 2017

\section{References}

1. Carlsson G, Huss-Danell K. Nitrogen fixation in perennial forage legumes in the field. Plant Soil. 2003;253:353-72.

2. Peoples MB, Brockwell J, Hunt JR, Swan AD, Watson L, Hayes RC, Li GD, Hackney B, Nuttall JG, Davies SL, Fillery IRP. Factors affecting the potential contributions of $\mathrm{N}_{2}$ fixation by legumes in Australian pasture systems. Crop Pasture Sci. 2012;63:759-86.

3. Unkovich MJ, Baldock J, Peoples MB. Prospects and problems of simple linear models for estimating symbiotic $\mathrm{N}_{2}$ fixation by crop and pasture legumes. Plant Soil. 2010;329:75-89.

4. UNDP 2015 [http://www.undp.org/content/undp/en/home/sdgoverview/ post-2015-development-agenda.html]. Accessed 22 Sept 2017.

5. Béna G, Lyet A, Huguet T, Olivieri I. Medicago-Sinorhizobium symbiotic specificity evolution and the geographic expansion of Medicago. J Evol Biol. 2005;18:1547-58.

6. Rome S, Fernandez MP, Brunel B, Normand P, Cleyet-Marel JC. Sinorhizobium medicae sp. nov., isolated from annual Medicago spp. Int J Syst Bacteriol. 1996;46:972-80.

7. Jones KM, Kobayashi H, Davies BW, Taga ME, Walker GC. How rhizobial symbionts invade plants: the Sinorhizobium-Medicago model. Nature Rev Microbiol. 2007:5:619-33.

8. Jordan DC. Reduction of the nodulation barrier in Medicago laciniata by alteration of the root temperature. Plant Soil. 1981;61:93-111.

9. Small E: Alfalfa and relatives: Evolution and classification of Medicago. Ottawa: NRC Research Press; 2010.

10. Young RR, Croft PH, Sandral GA. Variation in flowering times and agronomic characteristics of Medicago laciniata (L.) miller collected from diverse locations in new South Wales. Aust J Exp Agric. 1992;32:59-63.

11. Yousfi N, Sihem N, Ramzi A, Abdelly C. Growth, photosynthesis and water relations as affected by different drought regimes and subsequent recovery in Medicago laciniata (L.) populations. J Plant Biol. 2016;59:33-43.

12. Brockwell J, Hely FW. Symbiotic characteristics of Rhizobium meliloti: an appraisal of the systematic treatment of nodulation and nitrogen fixation interactions between hosts and rhizobia of diverse origins. Aust J Agr Econ. 1966;17:885-9.

13. Villegas MDC, Rome S, Mauré L, Domergue O, Gardan L, Bailly X, CleyetMarel J-C, Brunel B. Nitrogen-fixing sinorhizobia with Medicago laciniata constitute a novel biovar (bv. Medicaginis) of S. meliloti. Syst Appl Microbiol. 2006;29:526-38.

14. Barran LR, Bromfield ES, Brown DC. Identification and cloning of the bacterial nodulation specificity gene in the Sinorhizobium meliloti-Medicago laciniata symbiosis. Can J Microbiol. 2002;48:765-71.

15. van Berkum P, Badri Y, Elia P, Aouani ME, Eardly BD. Chromosomal and symbiotic relationships of rhizobia nodulating Medicago truncatula and $M$. laciniata. Appl Environ Microbiol. 2007;73:7597-604.

16. Mnasri B, Badri Y, Saïdi S, de Lajudie P, Mhamdi R. Symbiotic diversity of Ensifer meliloti strains recovered from various legume species in Tunisia. Syst Appl Microbiol. 2009;32:583-92.

17. Reeve W, Ardley J, Tian R, Eshragi L, Yoon JW, Ngamwisetkun P, Seshadri R, Ivanova NN, Kyrpides NC. A genomic encyclopedia of the root nodule bacteria: assessing genetic diversity through a systematic biogeographic survey. Stand Genomic Sci. 2015;10:14.

18. Seshadri R, Reeve WG, Ardley JK, Tennessen K, Woyke T, Kyrpides NC, Ivanova NN. Discovery of novel plant interaction determinants from the genomes of 163 root nodule bacteria. Sci Rep. 2015;5:16825.

19. Howieson JG, Dilworth MJ, editors. Working with Rhizobia. Canberra, Australia: Australian Centre for International Agricultural Research (ACIAR); 2016. 
20. Bailly X, Olivieri I, Brunel B, Cleyet-Marel JC, Béna G. Horizontal gene transfer and homologous recombination drive the evolution of the nitrogen-fixing symbionts of Medicago species. J Bacteriol. 2007;189:5223-36.

21. Delajudie P, Willems A, Pot B, Dewettinck D, Maestrojuan G, Neyra M, Collins MD, Dreyfus B, Kersters K, Gillis M. Polyphasic taxonomy of rhizobia: emendation of the genus Sinorhizobium and description of Sinorhizobium meliloti comb. nov., Sinorhizobium saheli sp. nov., and Sinorhizobium teranga sp. nov. Int I Syst Bacteriol. 1994:44:715-33.

22. Young JM. The genus name Ensifer Casida 1982 takes priority over Sinorhizobium Chen et al. 1988, and Sinorhizobium morelense Wang et al. 2002 is a later synonym of Ensifer adhaerens Casida 1982. Is the combination "Sinorhizobium adhaerens" (Casida 1982) Willems et al. 2003 legitimate? Request for an opinion. Int J Syst Evol Microbiol. 2003:53:2107-10.

23. Merabet C, Martens M, Mahdhi M, Zakhia F, Sy A, Le Roux C, Domergue O, Coopman R, Bekki A, Mars M, et al. Multilocus sequence analysis of root nodule isolates from Lotus arabicus (Senegal), Lotus creticus, Argyrolobium uniflorum and Medicago sativa (Tunisia) and description of Ensifer numidicus sp nov and Ensifer garamanticus sp nov. Int J Syst Evol Microbiol. 2010;60:664-74

24. Reddy TBK, Thomas AD, Stamatis D, Bertsch J, Isbandi M, Jansson J, Mallajosyula J, Pagani I, Lobos EA, Kyrpides NC. The genomes OnLine database (GOLD) v.5: a metadata management system based on a four level (meta)genome project classification. Nucleic Acids Res. 2015;43:D1099-106.

25. Markowitz VM, Chen IM, Palaniappan K, Chu K, Szeto E, Pillay M, Ratner A, Huang J, Woyke T, Huntemann M, et al. IMG 4 version of the integrated microbial genomes comparative analysis system. Nucleic Acids Res. 2014;42:D560-7.

26. van Berkum P. Evidence for a third uptake hydrogenase phenotype among the soybean bradyrhizobia. Appl Environ Microbiol. 1990;56:3835-41.

27. Bennett S. Solexa Ltd. Pharmacogenomics. 2004:5:433-8.

28. Joint Genome Institute website [http://jgi.doe.gov/]. Accessed 22 Sept 2017.

29. Zerbino DR, Birney E. Velvet: algorithms for de novo short read assembly using de Bruijn graphs. Genome Res. 2008;18:821-9.

30. GitHub - Ih3/wgsim: Reads simulator [https://github.com/lh3/wgsim]. Accessed 22 Sept 2017.

31. Gnerre S, MacCallum I, Przybylski D, Ribeiro FJ, Burton JN, Walker BJ, Sharpe T, Hall G, Shea TP, Sykes S, et al. High-quality draft assemblies of mammalian genomes from massively parallel sequence data. Proc Natl Acad Sci U S A. 2011;108:1513-8.

32. Hyatt D, Chen GL, Locascio PF, Land ML, Larimer FW, Hauser L. Prodigal: prokaryotic gene recognition and translation initiation site identification. BMC Bioinformatics. 2010;11:119.

33. Huntemann M, Ivanova NN, Mavromatis K, Tripp HJ, Páez-Espino D, Palaniappan K, Szeto E, Pillay M, Chen IM, Pati A, et al. The standard operating procedure of the DOE-JGI microbial genome annotation pipeline (MGAP v.4). Stand Genomic Sci. 2015;10:86.

34. Chen IM, Markowitz VM, Chu K, Anderson I, Mavromatis K, Kyrpides NC, Ivanova NN. Improving microbial genome annotations in an integrated database context. PLoS One. 2013;8:e54859.

35. Lowe TM, Eddy SR. tRNAscan-SE: a program for improved detection of transfer RNA genes in genomic sequence. Nucleic Acids Res. 1997;25:955-64.

36. Pruesse E, Quast C, Knittel K, BdM F, Ludwig W, Peplies J, Glöckner FO. SILVA: a comprehensive online resource for quality checked and aligned ribosomal RNA sequence data compatible with ARB. Nucleic Acids Res. 2007:35:7188-96.

37. Nawrocki EP, Eddy SR. Infernal 1.1: 100-fold faster RNA homology searches. Bioinformatics. 2013;29:2933-5.

38. Markowitz VM, Mavromatis K, Ivanova NN, Chen IM, Chu K, Kyrpides NC IMG ER: a system for microbial genome annotation expert review and curation. Bioinformatics. 2009;25:2271-8.

39. Varghese NJ, Mukherjee S, Ivanova N, Konstantinidis KT, Mavrommatis K, Kyrpides NC, Pati A. Microbial species delineation using whole genome sequences. Nucleic Acids Res. 2015:43:6761-71.

40. Korotkov KV, Sandkvist M, Hol WG. The type II secretion system: biogenesis, molecular architecture and mechanism. Nature Rev Microbiol. 2012;10:336-51.

41. Krysciak D, Orbegoso MR, Schmeisser C, Streit WR. Molecular keys to broad host range in Sinorhizobium fredii NGR234, USDA257 and HH103. In: De Bruijn FJ, editor. Biological nitrogen fixation. Volume 2. Hoboken NJ: Wiley-Blackwell; 2015. p. 325-36.

42. Schmeisser C, Liesegang H, Krysciak D, Bakkou N, Le Quéré A, Wollherr A, Heinemeyer I, Morgenstern B, Pommerening-Röser A, Flores $M$, et al. Rhizobium sp. strain NGR234 possesses a remarkable number of secretion systems. Appl Environ Microbiol. 2009;75:4035-45.
43. Costa TR, Felisberto-Rodrigues C, Meir A, Prevost MS, Redzej A, Trokter M, Waksman G. Secretion systems in gram-negative bacteria: structural and mechanistic insights. Nature Rev Microbiol. 2015;13:343-59.

44. Arndt D, Grant JR, Marcu A, Sajed T, Pon A, Liang Y, Wishart DS. PHASTER: a better, faster version of the PHAST phage search tool. Nucleic Acids Res. 2016;44:W16-21.

45. Tiwari RP, Reeve WG, Dilworth MJ, Glenn AR. An essential role for actA in acid tolerance of Rhizobium meliloti. Microbiology. 1996;142:601-10.

46. Tiwari RP, Reeve WG, Dilworth MJ, Glenn AR. Acid tolerance in Rhizobium meliloti strain WSM419 involves a two-component sensor-regulator system. Microbiology. 1996;142:1693-704.

47. Tiwari RP, Reeve WG, Fenner BJ, Dilworth MJ, Glenn AR, Howieson JG. Probing for pH-regulated genes in Sinorhizobium medicae using transcriptional analysis. J Mol Microbiol Biotechnol. 2004;7:133-9.

48. Reeve WG, Dilworth MJ, Tiwari RP, Glenn AR. Regulation of exopolysaccharide production in Rhizobium leguminosarum biovar viciae WSM710 involves exoR. Microbiology. 1997;143:1951-8.

49. Reeve WG, Tiwari RP, Guerreiro N, Stubbs J, Dilworth MJ, Glenn AR, Rolfe BG, Djordjevic MA, Howieson JG. Probing for pH-regulated proteins in Sinorhizobium medicae using proteomic analysis. J Mol Microbiol Biotechnol. 2004;7:140-7.

50. Reeve WG, Tiwari RP, Kale NB, Dilworth MJ, Glenn AR. ActP controls copper homeostasis in Rhizobium leguminosarum bv. Viciae and Sinorhizobium meliloti preventing low pH-induced copper toxicity. Mol Microbiol. 2002;43:981-91.

51. Reeve WG, Tiwari RP, Wong CM, Dilworth MJ, Glenn AR. The transcriptional regulator gene phrR in Sinorhizobium meliloti WSM419 is regulated by low pH and other stresses. Microbiology. 1998;144:3335-42.

52. Glenn AR, Reeve WG, Tiwari RP, Dilworth MJ. Acid tolerance in root nodule bacteria. In: Chadwick DJ, Cardew G, editors. Bacterial response to $\mathrm{pH}$ Novartis Foundation symposium volume 221. 1999/04/20 edition. London: Wiley Publishing; 1999. p. 112-6.

53. Reeve WG, Brau L, Castelli J, Garau G, Sohlenkamp C, Geiger O, Dilworth MJ, Glenn AR, Howieson JG, Tiwari RP. The Sinorhizobium medicae WSM419 IpiA gene is transcriptionally activated by FsrR and required to enhance survival in lethal acid conditions. Microbiology. 2006;152:3049-59.

54. Lerouge $P$, Roche $P$, Faucher $C$, Maillet $F$, Truchet $G$, Promé JC, Dénarié J. Symbiotic host-specificity of Rhizobium meliloti is determined by a sulphated and acylated glucosamine oligosaccharide signal. Nature. 1990;344:781-4.

55. Dénarié J, Debellé F, Promé JC. Rhizobium lipo-chitooligosaccharide nodulation factors: signaling molecules mediating recognition and morphogenesis. Annu Rev Biochem. 1996;65:503-35.

56. Schultze M, Staehelin C, Röhrig H, John M, Schmidt J, Kondorosi E, Schell J, Kondorosi A. In vitro sulfotransferase activity of Rhizobium meliloti NodH protein: lipochitooligosaccharide nodulation signals are sulfated after synthesis of the core structure. Proc Natl Acad Sci U S A. 1995;92:2706-9.

57. Schwedock JS, Liu C, Leyh TS, Long SR. Rhizobium meliloti NodP and NodQ form a multifunctional sulfate-activating complex requiring GTP for activity. J Bacteriol. 1994;176:7055-64.

58. Ardourel M, Lortet G, Maillet F, Roche P, Truchet G, Promé JC, Rosenberg C. In Rhizobium meliloti, the operon associated with the nod box $\mathrm{n} 5$ comprises nodL, noeA and noeB, three host-range genes specifically required for the nodulation of particular Medicago species. Mol Microbiol. 1995;17:687-99.

59. Demont N, Debellé F, Aurelle H, Dénarié J, Promé JC. Role of the Rhizobium meliloti nodF and nodE genes in the biosynthesis of lipo-oligosaccharidic nodulation factors. J Biol Chem. 1993:268:20134-42.

60. Honma MA, Asomaning M, Ausubel FM. Rhizobium meliloti nodD genes mediate host-specific activation of nodABC. J Bacteriol. 1990;172:901-11.

61. Tamura K, Stecher G, Peterson D, Filipski A, Kumar S. MEGA6: molecular evolutionary genetics analysis version 6.0. Mol Biol Evol. 2013;30:2725-9.

62. Nicholas KB, Nicholas HB, Deerfield DW. GeneDoc: analysis and visualization of genetic variation. EMBnew News. 1997;4:14.

63. van Berkum P, Terefework Z, Paulin L, Suomalainen S, Lindström K, Eardly BD. Discordant phylogenies within the rrn loci of rhizobia. J Bacteriol. 2003;185:2988-98.

64. Felsenstein J. Confidence limits on phylogenies: an approach using the bootstrap. Evolution. 1985;39:783-91.

65. Field D, Garrity G, Gray T, Morrison N, Selengut J, Sterk P, Tatusova T, Thomson N, Allen M, Angiuoli SV, et al. Towards a richer description of our complete collection of genomes and metagenomes "minimum information about a genome sequence" (MIGS) specification. Nature Biotechnol. 2008;26:541-7.

66. Field D, Amaral-Zettler L, Cochrane G, Cole JR, Dawyndt P, Garrity GM, Gilbert J, Glöckner FO, Hirschman L, Karsch-Mizrachi I, et al. The genomic standards consortium. PLoS Biol. 2011;9:e1001088. 
67. Woese CR, Kandler O, Wheelis ML. Towards a natural system of organisms: proposal for the domains Archaea, bacteria, and Eucarya. Proc Natl Acad Sci U S A. 1990;87:4576-9.

68. Garrity GM, Bell JA, Lilburn T. In: Garrity GM, Brenner DJ, Kreig NR, Staley JT, editors. Phylum XIV. Proteobacteria phyl. Nov. in Bergey's manual of systematic bacteriology. Volume 2. Second edition. New York: Springer - Verlag; 2005. p. 1.

69. Garrity GM, Bell JA, Lilburn T. In: Garrity GM, Brenner DJ, Kreig NR, Staley JT, editors. Class I. Alphaproteobacteria class. In Bergey's manual of systematic bacteriology. Second edition. New York: Springer - Verlag; 2005.

70. Euzéby J. Validation list no. 107. List of new names and new combinations previously effectively, but not validly, published. Int I Syst Evol Microbiol. 2006;56:1-6.

71. Kuykendall LD. In: Garrity GM, Brenner DJ, Kreig NR, Staley JT, editors. Order VI. Rhizobiales ord. Nov. in Bergey's manual of systematic bacteriology. Second edition. New York: Springer - Verlag; 2005. p. 324

72. Skerman VBD, McGowan V, Sneath PHA. Approved lists of bacterial names. Int J Syst Bacteriol. 1980;30:225-420.

73. Conn HJ. Taxonomic relationships of certain non-sporeforming rods in soil. J Bacteriol. 1938;36:320-1.

74. Casida LE. Ensifer adhaerens gen. Nov., sp. nov.: a bacterial predator of bacteria in soil. Int J Syst Evol Microbiol. 1982;32:339-45.

75. Biological Agents: Technical rules for biological agents [http://www.baua.de/ en/Topics-from-A-to-Z/Biological-Agents/TRBA/TRBA.html]. Accessed $22 \mathrm{Sept}$ 2017.

76. Ashburner M, Ball CA, Blake JA, Botstein D, Butler H, Cherry JM, Davis AP, Dolinski K, Dwight SS, Eppig JT, et al. Gene ontology: tool for the unification of biology. The Gene Ontology Consortium Nature Genet. 2000;25:25-9.

77. Guide to GO Evidence Codes [http://geneontology.org/page/guide-goevidence-codes]. Accessed 22 Sept 2017.

78. Locus Tag [https:/www.ncbi.nlm.nih.gov/bioproject/?term=A3CA]. Accessed 22 Sept 2017.

79. GOLD ID for Ensifer meliloti Mlalz-1 [https://gold.jgi.doe.gov/projects?id= Gp0010229]. Accessed 22 Sept 2017.

\section{Submit your next manuscript to BioMed Central and we will help you at every step:}

- We accept pre-submission inquiries

- Our selector tool helps you to find the most relevant journal

- We provide round the clock customer support

- Convenient online submission

- Thorough peer review

- Inclusion in PubMed and all major indexing services

- Maximum visibility for your research

Submit your manuscript at www.biomedcentral.com/submit

) Biomed Central 